\title{
Occupational Safety Control is a Way to Reduce Workplace Injuries at Manufacturing Plants
}

\author{
Nikolay Ligkiy
}

\begin{abstract}
This article analysis causes of injury at the manufacturing plants that have a high level of risk and explains a need to control the occupational safety and comply with work procedures. An author provides quantitative and qualitative performance indicators at all levels. The article also explains a mathematical model of observations and evaluates its accuracy and adequacy in the context of the decision-making conditions. Finally, there are findings on correlation between the number of detected safety violations and experience of an observer.
\end{abstract}

Index Terms: occupational injuries, occupational safety, control, observer, observer experience

\section{INTRODUCTION}

The level of occupational injuries depends on many factors. One of the most important factors is the quality of control over the safety and observance of operating modes, both the workers themselves and technological processes. The main quantitative indicators, which assess the effectiveness of monitoring activities at all levels, are indicators of occupational injuries, namely: the number of accidents with severe consequences; - the number of fatally injured workers; the number of victims of accidents; financial costs associated with the implementation of occupational safety measures and a number of other indicators. In the construction of the dependence of the level of injury on the set of indicators of control activities provides for the presence of an employee-observer and the quality of the work performed by him. Particularly relevant are safety checks when adjusting high-frequency products, for example, such as [1].

\section{RESEARCH METHOD}

To solve this problem, it is necessary to determine the initial data array and the algorithm for constructing a mathematical model, to build a model, to assess its accuracy and adequacy to the decision-making conditions, to find the minimum value taking into account the restrictions [2].

In General, the observation model will look like:

$$
Y=F(X)=a_{0}+\sum_{i=1}^{n} a_{i} X_{i}+\sum_{i=1}^{n} \sum_{j=1}^{n} a_{i j} X_{i} X_{j}+\ldots
$$

where - observation indicator;

, - factors (input variables);

, , - the constant coefficients of the model;

- number of factors to consider.

Model (1) is used in cases where it is not known in advance that the modelled indicator, except for factors that are directly

Revised Manuscript Received on July 09, 2019.

Nikolay Ligkiy, (Corresponding Author) MIREA - Russian Technological University, 78, Vernadskogo pr., Moscow, 119454 Russia, Email: legki@mirea.ru taken into account in the modeling, is also affected by many other factors, the influence of which can not be taken into account or eliminated. A number of factors, whose influence must be considered impossible to eliminate, determines the presence in model (1) member of . The construction of the model consists in the calculation of a set of constant coefficients of the polynomial, which establish the relationship between the modeled indicator and a set of factors. An example of the source data is shown in tab. 1.

Table 1. Array of initial data for modeling

\begin{tabular}{|c|c|c|c|}
\hline Indicator & \multicolumn{3}{|c|}{ Factors } \\
\hline$Y$ & $X_{1}$ & $\ldots$ & $X_{n}$ \\
\hline$y_{1}$ & $x_{11}$ & $\ldots$ & $x_{1 n}$ \\
\hline$\ldots$ & $\ldots$ & $\ldots$ & $\ldots$ \\
\hline$y_{m}$ & $x_{m 1}$ & $\ldots$ & $x_{m n}$ \\
\hline
\end{tabular}

In the table: the first column is a simulated indicator $Y$, the other columns are factors $X_{1}, \ldots, X_{n}$, which affect the predicted indicator $Y, n$ - the number of factors that are taken into account, $m$ - the number of indicators.

In general, there is no strict correspondence between the values of the indicator and the factor in each case. The model excludes those factors that do not have a significant impact on the studied indicator. To do this, it is necessary to calculate the coefficients of pair correlation $X_{Y_{i} R}$, which establish the density of the relationship between the indicator $Y$ and factors $X_{i}$. The correlation coefficient between $Y$ and factor $X_{i}$ can be calculated by the formula:

$$
R_{X_{i} Y}=\frac{\sum_{j=1}^{m}\left(x_{i j}-\overline{x_{i}}\right) \cdot\left(y_{j}-\bar{y}\right)}{\sqrt{\left(\sum_{j=1}^{m} x_{i j}-\bar{x}_{i}^{2}\right) \cdot\left(\sum_{j=1}^{m} y_{j}-\bar{y}^{2}\right)}}
$$

where $\bar{x}_{i}, \bar{y}$ - the average values of factor $X_{i}$ and indicator $Y$, which are determined respectively:

$$
\overline{x_{i}}=\frac{1}{m} \sum_{j=1}^{m} x_{i j}, \bar{y}=\frac{1}{m} \sum_{j=1}^{m} y_{j}
$$

The set of factors (Table 1) must be sorted in descending order of values of correlation coefficients $X_{Y_{i} R}$. 
The maximum permissible number of factors for mathematical models is $m-1$, that is, the first $m-1$ factors are selected from the sorted data set, the rest are excluded from further consideration. Construction of mathematical models is carried out by regression-correlation analysis. While built many models with iteratively increasing the number of members of the polynomial:

$$
\begin{gathered}
Y^{1}=a_{0}+a_{1} X_{1} \\
Y^{2}=a_{0}+a_{1} X_{1}+a_{2} X_{2} ; \\
\cdots \\
Y^{k}=a_{0}+\sum_{i=1}^{k} a_{i} X_{i}
\end{gathered}
$$

The accuracy of the approximation of each of the set of constructed models $\Delta_{A}^{i}$ estimated deviation of the actual and calculated values of the simulated indicator [\%]

$$
\Delta_{A}^{i}=100-\delta_{A}^{i}
$$

where $\delta_{A}^{i}$ - approximation error when using model $i$ :

$$
\delta_{A}^{i}=\frac{1}{m} \sum_{i=1}^{m}\left|\frac{y_{j}-y_{j}^{i}}{y_{j}}\right| \cdot 100 \%
$$

where $y_{i}, y_{j}^{i}(j=1, \ldots, m)$-accordingly, the actual and calculated value of the indicator, which is obtained using the model i of the subset $Y$.

The construction and study of the proposed mathematical models allows for in-depth comprehensive analysis of industrial injuries, as the most significant indicator of the state of industrial safety and labor protection in enterprises.

\section{RESULTS AND ANALYSIS}

As an effective option for assessing the level of professional competence of observers, an anonymous survey of specialists and heads of departments and services of labor protection of enterprises and heads of production departments was used. According to the results of the questionnaire data processing, the dependences presented in Fig. 1.

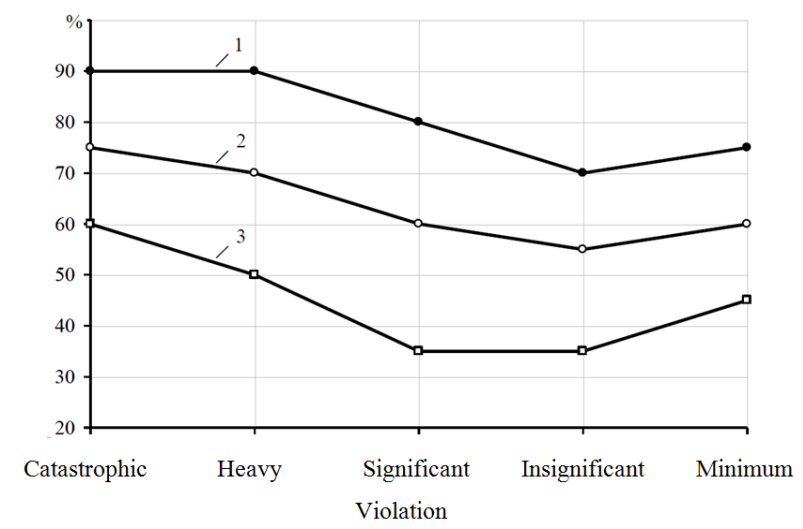

Figure. 1. Dependence of the percentage of violations detected on the severity of a dangerous event for controllers with different levels of their professional training: 1 - the highest; 2 - medium; 3 - low
Analysis of the literature, in particular [3-6], allowed to obtain average data on the number of days of disability as a result of accidents due to "Violation of labor and industrial discipline».

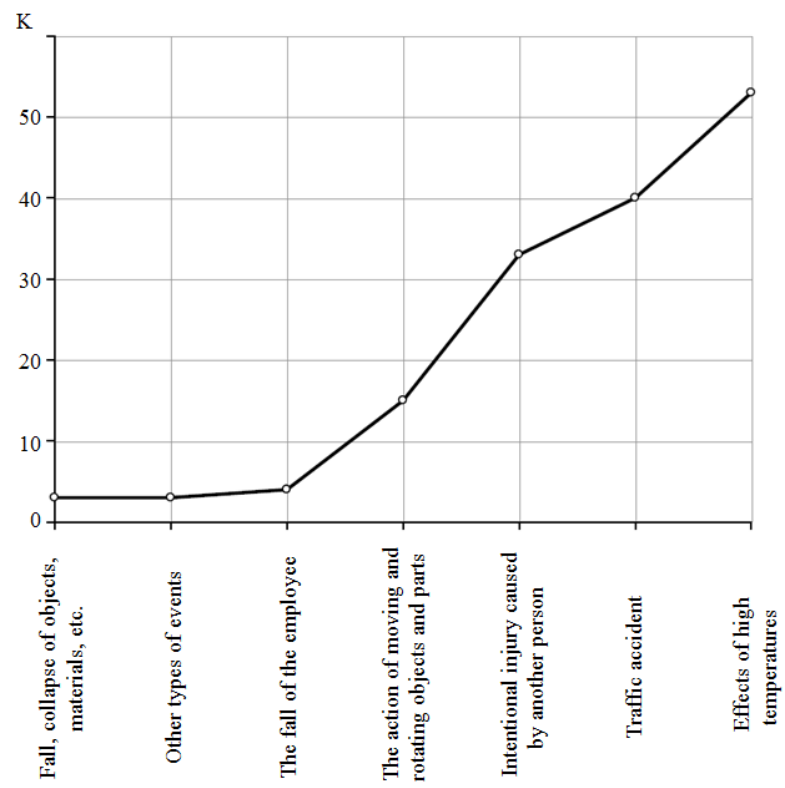

Figure 2. Dependence of the number of days of disability K as a result of accidents due to "Violation of labor and production discipline" on various types of accidents

\section{CONCLUSION}

The use of mathematical models of indicators in the implementation of management and monitoring activities in the field of labor protection allows to solve two main tasks: to identify and quantify the impact of factors on the simulated indicators and obtain the projected values of these indicators.

Practical use of the results allows to increase the validity of management decisions in the field of labor protection. The use of mathematical models becomes a tool for identifying and investigating situations that are determined by a set of values of factors and calculated values of the indicator. The results of calculations in accordance with the constructed models can be used in the development of recommendations for the planning of occupational safety measures and to justify the necessary funding for these activities.

\section{Conflict of Interest}

The authors declare no conflict of interest.

\section{REFERENCES}

1. N.M. Legkiy, I.V. Unchenko, "Formation Of The Direction Diagram In Phased Antenna Array" Rossiyskiy tekhnologicheskiy zhurnal (Russian Technological Journal). 2019; 7(2): 29-38. (in Russ.). DOI: $10.32362 / 2500-316 \mathrm{X}-2019-7-2-29-38$.

2. URL

https://rtj.mirea.ru/upload/medialibrary/763/RTZH 2201929 38.pdf

3. A.A. Pastushkov, V.K. Batovrin, "Selection Of Solutions For Designing Open Systems Based On Analysis Of Variants With Random Weights" Rossiyskiy tekhnologicheskiy zhurnal (Russian Technological Journal). 2018; 6(4): 78-88. (in Russ.).

4. URL 
https://rtj.mirea.ru/upload/medialibrary/bf5/RTZH $4 \quad 2018 \quad 78$ 88.pdf

5. M.L. Chipman, "Injury Control: A Guide To Research And Program Evaluation” American Journal of Epidemiology. 2001. V. 154. № 3. P. 285-a.

6. C. Peek-Asa , C. Zwerling, "Role Of Environmental Interventions In Injury Control And Prevention" Epidemiologic Reviews. 2003. V. 25. № 1. P. 77.

7. T. Virén, S. Saarakkala, J.S. Jurvelin, J. Töyräs, V. Tiitu, J. Puhakka, I. Kiviranta, "Ultrasound Evaluation Of Mechanical Injury Of Bovine Knee Articular Cartilage Under Arthroscopic Control" IEEE Transactions on Ultrasonics, Ferroelectrics, and Frequency Control. 2011. V. 58. № 1. P. 148-155.

8. V.B. Artemyev, A.B. Kilin, G.N. Shapovalenko, A.V. Osharov, S.N. Radionov, I.L. Kravchuk, "The Concept Of Advanced Control As A Means Of Significantly Reducing Injuries" Magazine "COAL", May, 2013, P. 82 -85.(in Russ.). 